\title{
Study of Upper Critical Magnetic Field of Superconducting $\mathrm{HoM}_{6} \mathrm{Se}_{8}$
}

\author{
Tadesse Desta1, Pooran Singh1, Gebregziabher Kahsay² \\ ${ }^{1}$ Department of Physics, College of Natural Science, Addis Ababa University, Addis Ababa, Ethiopia \\ ${ }^{2}$ Department of Physics, College of Science, Bahir Dar University, Bahir Dar, Ethiopia \\ Email: tad4jiu@gmail.com, psinghgbpup@yahoo.com, michige 90@yahoo.com
}

Received 25 April 2015; accepted 24 July 2015; published 27 July 2015

Copyright (C) 2015 by authors and Scientific Research Publishing Inc.

This work is licensed under the Creative Commons Attribution International License (CC BY).

http://creativecommons.org/licenses/by/4.0/

cc) (i) Open Access

\section{Abstract}

This work focuses on the study of mathematical aspects of upper critical magnetic field of superconducting $\mathrm{HoMo}_{6} \mathrm{Se}_{8}$. At zero external magnetic field, $\mathrm{HoMo}_{6} \mathrm{Se}_{8}$ was found to undergo a transition from the normal state to the superconducting state at $5.6 \mathrm{~K}$ and returned to a normal but magnetically ordered state between the temperature range of $0.3 \mathrm{~K}$ and $0.53 \mathrm{~K}$. The main objective of this work is to show the temperature dependence of the upper critical magnetic field of superconducting $\mathrm{HoMo}_{6} \mathrm{Se}_{8}$ by using the Ginzburg-Landau (GL) phenomenological Equation. We found the direct relationship between the GL coherence length $\left(\xi_{G L}\right)$ and penetration depth $\left(\lambda_{G L}\right)$ with temperature. From the GL Equations and the results obtained for the GL coherence length, the expression for upper critical magnetic field $\left(\mathrm{H}_{c 2}\right)$ is obtained for the superconducting $\mathrm{HoMo}_{6} \mathrm{Se}_{8}$. The result is plotted as a function of temperature. The graph shows the linear dependence of upper critical magnetic field $\left(H_{c 2}\right)$ with temperature $(\mathrm{T})$ and our finding is in agreement with experimental observations.

\section{Keywords}

Ginzburg-Landau Equation, Upper Critical Magnetic Field, $\mathrm{HoMo}_{6} \mathrm{Se}_{8}$

\section{Introduction}

Superconductivity is a phenomenon that occurs at very low temperatures. Every superconductor has a transition temperature $\left(T_{c}\right)$ below which it superconducts and above which it is a normal metal [1]. In the superconducting state, the material has no electrical resistance and thus conducts electricity without losses. On the other hand, in the normal state, the material does have resistance and the flow of electric current accompanies with the development of heat and the dissipation of energy [1]. 
The era of low-temperature physics began in 1908 when the Dutch physicist Heike Kamerlingh Onnes first liquefied helium which boiled at $4.2 \mathrm{~K}$ at standard pressure. Three years later, in 1911, Kamerlingh Onnes discovered the phenomenon of superconductivity while studying the resistivity of metals at low temperatures [1].

Most of the fundamental properties of superconductors vary from material to material. The superconducting state, as any state of matter, has its own basic properties. So, any superconductor independent of the mechanism of superconductivity and the material will exhibit these properties. The basic properties of the superconducting state are zero resistance, Meissner effect, magnetic flux quantization, Josephson effects, the BCS theory, Cooper pair, appearance of an energy gap in elementary excitation energy spectrum, Isotope effect and the proximity effect. Every superconducting transition is marked by a jump in specific heat. In the mixed state, the behavior of type-II superconductors has the same pattern [3] [4].

$\mathrm{HoMo}_{6} \mathrm{Se}_{8}$ compound is building blocks of the Chevrel-phase crystal structure [5]. The discovery in 1984 of the new superconducting $\mathrm{HoMo}_{6} \mathrm{Se}_{8}$ gave rise to a renewed interest in the interplay of magnetism and superconductivity. From the experimental results, a magnetic phase transition $\left(T_{n}=0.53 \mathrm{~K}\right)$ to a long-period magnetic states has been observed via neutron scattering in the superconductor $\mathrm{HoMo}_{6} \mathrm{Se}_{8}\left(T_{c}=5.6 \mathrm{~K}\right)$ [6]. The characteristic wave vector $\left(q_{c}\right)$ is strongly temperature dependent and there is no observation of higher-order satellites.

The rare occurrence of ferromagnetism, as found in $\mathrm{HoMo}_{6} \mathrm{Se}_{8}$ and $\mathrm{HoMo}_{6} \mathrm{Se}_{8}$, revealed the strongly competitive nature of these two cooperative phenomena in the form of long-wavelength at low oscillatory magnetic temperature $(<1 \mathrm{~K})$ and a ferromagnetic lock-in transition that quenched the superconductivity. No sign of reentrant behavior (in zero field) was observed down to $0.04 \mathrm{~K}$ [6] [7]. Ternary rare-earth superconductors which display a propensity for ferromagnetism have received considerable experimental as well as theoretical attention recently. In the case of the new superconductors, $\mathrm{HoMo}_{6} \mathrm{Se}_{8}$ was observed despite the presence of the holmium moment of the rare earth ion in each unit cell. These compounds did not destroy the property of superconductivity at low temperature. In $\mathrm{HoMo}_{6} \mathrm{Se}_{8}$, the domain coexistence phase survives till $T=0.3 \mathrm{~K}$ (the exchange interaction is weaker and the coexistence persists down to $T=0.3 \mathrm{~K}$ ) [8]. In addition to the superconducting and ferromagnetic domains, a coexistence region is observed in $\mathrm{HoMo}_{6} \mathrm{Se}_{8}$ in which the superconducting state coexists with a long range modulated magnetic order in a narrow region above the reentrant temperature $T_{c 2}$.

The upper critical magnetic field is a very important magnetic superconductivity parameter. Therefore, starting from its discovery as a superconducting material, experiments are carried out to analysis the upper critical magnetic field $\left(\mathrm{H}_{c 2}\right)$ of $\mathrm{HoMo}_{6} \mathrm{Se}_{8}$.

According to magnetization measurements, on both poly and single crystalline samples of the ferromagnetic superconducting $\mathrm{HoMo}_{6} \mathrm{Se}_{8}$, the upper critical magnetic field is a turning point from the superconducting state to the normal state. The first-order phase transition is the inter changing point of the superconducting state to the normal state observed when the external field $\mathrm{H}$ is applied parallel to the magnetically easy axis (a axis of the hexagonal-rhombohedral crystal lattice structure).

The critical magnetic field in type-I superconductor is the inter changing point of superconducting state into normal state. From Maxwell's Equation, $\nabla X \boldsymbol{E}=-\frac{\partial B}{\partial t}$, when the magnetic field is frozen, the field is expelled from the interior of the superconductors, otherwise superconductivity will be destroyed by a critical magnetic field $\left(H_{c}\right)$, such that

$$
H_{c}(T)=H_{c(0)}\left[1-\left(\frac{T}{T_{c}}\right)^{2}\right]
$$

Equation (1) yields the expression of thermodynamic critical magnetic field $\left(H_{c}(T)\right)$ [9].

\section{Mathematical Formulations to Find the Upper Critical Magnetic Field of $\mathrm{HoMo}_{6} \mathrm{Se}_{8}$}

\subsection{The Basic Ginzburg-Landau Theory}

Ginzburg-Landau (GL) theory is a mathematical theory used to describe superconductivity. Ginzburg-Landau (GL) theory is used to explain the difference between Type-I and Type-II superconductors and enables the calculation of two critical magnetic fields $H_{c 1}$ and $H_{c 2}$ [10]. Ginzburg-Landau theory was derived from the BCS microscopic theory by Lev Gorkov, showing that it also appears in some limit of microscopic theory and apply- 
ing microscopic interpretation of all its parameters.

The basic postulate of GL is that if $\psi$ is small and varies slowly in space, the free-energy density $\left(F_{s}(r)\right)$ can be expanded in a series of the form:

$$
F_{s}=F_{n}+\alpha|\psi|^{2}+\frac{\beta}{2}|\psi|^{4}+\frac{1}{2 m^{*}}\left|\left(-i \hbar \nabla-\frac{e^{*}}{c} \boldsymbol{A}\right) \psi\right|^{2}+\frac{|H|^{2}}{8 \pi}
$$

where $\alpha$ and $\beta$ are phenomenological parameters, ( $\beta$ is positive and the sign of $\alpha$ is temperature dependent), $m^{*}=2 m$ is an effective mass, $e^{*}=q^{*}=2 e$ is the charge of an electron, $\boldsymbol{A}$ is the magnetic vector potential and $\boldsymbol{B}=\nabla X \boldsymbol{A}$ is the magnetic field [5] [10].

If $\psi=0$, Equation (2) reduces to the free energy of the normal state; $F_{s}=F_{n}+\frac{|H|^{2}}{8 \pi}$.

Now, by minimizing the free energy with respect to fluctuations in the order parameter and the vector potential, one arrives at the Ginzburg-Landau Equations given by:

$$
\alpha \psi+\beta|\psi|^{2} \psi+\frac{1}{2 m^{*}}\left(-i \hbar \nabla-\frac{e^{*}}{c} \boldsymbol{A}\right)^{2} \psi=0
$$

The current density from the Hamilton's energy of particles is given by;

$$
\begin{gathered}
H=\frac{1}{2} m^{*} v_{d}^{2}=\frac{1}{2 m^{*}}\left[\boldsymbol{p}-\frac{e^{*}}{c} \boldsymbol{A}\right]^{2} \\
v_{d}^{2}=\frac{1}{m^{* 2}}\left[\boldsymbol{p}-\frac{e^{*}}{c} \boldsymbol{A}\right]^{2} \\
v_{d}=\frac{1}{m^{*}}\left[\boldsymbol{p}-\frac{e^{*}}{c} \boldsymbol{A}\right]
\end{gathered}
$$

where $\boldsymbol{p}=-i \hbar \nabla$

$$
\begin{gathered}
v_{d}=\frac{1}{m^{*}}\left[-i \hbar \nabla-\frac{e^{*}}{c} \boldsymbol{A}\right] \\
\boldsymbol{J}_{s}=e^{*} n_{s} v_{d}
\end{gathered}
$$

where $n_{s}=\psi(r)^{*} \psi(r)$

$$
\begin{gathered}
\boldsymbol{J}_{s}=\frac{e^{*}}{m^{*}}\left[-i \hbar \nabla-\frac{e^{*}}{c} \boldsymbol{A}\right] \psi(r)^{*} \psi(r) \\
\boldsymbol{j}_{s}=\frac{e^{*}}{m^{*}}\left[\psi^{*}\left(-i \hbar \nabla-\frac{e^{*}}{c} \boldsymbol{A}\right) \psi+\psi\left(-i \hbar \nabla-\frac{e^{*}}{c} \boldsymbol{A}\right) \psi^{*}\right] \\
\boldsymbol{j}_{s}=\frac{e^{*}}{m^{*}}\left[\psi^{*}\left(\frac{\hbar}{i} \nabla-\frac{e^{*}}{c} \boldsymbol{A}\right) \psi+\psi\left(\frac{\hbar}{i} \nabla-\frac{e^{*}}{c} \boldsymbol{A}\right) \psi^{*}\right]
\end{gathered}
$$

where $\boldsymbol{j}_{s}$ is supercurrent density.

Equation (3) determines the order parameter $\psi$ based on the applied magnetic field and Equation (5) yields the superconducting current density. The Ginzburg-Landau Equation provides complete information about the superconducting state $\psi(r)$ that gives the spatial distribution of the Cooper pair density taking into account a possible variation in their concentration, where as $\boldsymbol{A}(r)$ describes the local distribution of the magnetic field 
in the superconductor.

In the absence of external magnetic field (at free surface), there will not be superconducting current(current flow) and the Equation for $\psi$ becomes:

$$
F_{s}-F_{n}=\alpha \psi+\beta|\psi|^{2} \psi
$$

This Equation has a trivial solution $\psi=0$ and it corresponds to normal state of $T>T_{c}$. Below superconducting transition temperature $\left(T_{c}\right)$, Equation (6) is expected to have a non-trivial solution (i.e. $\left.\psi \neq 0\right)$ and the Equation can be rearranged into:

$$
|\psi|^{2}=-\frac{\alpha}{\beta}
$$

If the second part of Equation (3) is positive, then there is a non zero solution for $\psi$ and this can be achieved by assuming the temperature dependence of $\alpha$ such that $\alpha(T)=\alpha_{0}\left(T-T_{c}\right)$ with $\frac{\alpha_{0}}{\beta}>0$ and $n_{s} \propto\left(T_{c}-T\right)$. For $T>T_{c}$, the expression $\frac{\alpha(T)}{\beta}$ is positive and the second part of Equation (3) is negative and only $\psi=0$ solves the Ginzburg-Landau Equation. For $T<T_{c}$, the second part of Equation (3) is positive and there is a non-trivial solution for $\psi$. Thus Equation (7) can be expressed as:

$$
\begin{gathered}
|\psi|^{2}=\frac{\alpha_{0}\left(T_{c}-T\right)}{\beta} \\
|\psi|=\left(\frac{\alpha_{0}\left(T_{c}-T\right)}{\beta}\right)^{\frac{1}{2}}
\end{gathered}
$$

Equation (8) yields Ginzburg Landau order parameter [5] [11].

\subsection{Calculation of Ginzburg-Landau Coherence Length}

The Ginzbrug-Landau coherence length $\left(\xi_{G L}\right)$ is a measure of the distance in the superconducting electron concentration that can not change drastically in a spatially-varying magnetic field. The Ginzbrug-Landau coherence length $\left(\xi_{G L}\right)$ is a temperature-dependent as well as a material dependent quantity. In the case of absence of the magnetic vector potential, Equation (3) reduces to:

$$
\alpha \psi+\beta|\psi|^{3}+\frac{1}{2 m^{*}}(-i \hbar \nabla)^{2} \psi=0
$$

Now, let us consider a wave function that varies only in the z-direction with zero applied magnetic field. In this case, the first GL Equation is one dimensional. i.e,

$$
\alpha \psi+\beta|\psi|^{3}-\frac{\hbar^{2}}{2 m^{*}} \frac{\mathrm{d}^{2} \psi}{\mathrm{d} x^{2}}=0
$$

Assuming $\psi$ is real and neglecting the term $\beta|\psi|^{3}$ in comparison with $\alpha$, Equation (10), becomes:

$$
\alpha \psi=-\frac{\hbar^{2}}{2 m^{*}} \frac{\mathrm{d}^{2} \psi}{\mathrm{d} x^{2}}
$$

For the plane wave function, the solution of Equation (11) is in the form of,

$$
\psi(x)=\mathrm{e}^{\left(\frac{i x}{\xi_{G L}}\right)}=\exp \left(\frac{i x}{\xi_{G L}}\right)
$$

Substituting the value of plane wave function into Equation (11) (in terms of $(\psi(x))$ ), we get, 


$$
\begin{gathered}
\alpha\left[\exp \left(\frac{i x}{\xi_{G L}}\right)\right]=-\frac{\hbar^{2}}{2 m^{*}} \frac{\mathrm{d}^{2}}{\mathrm{~d} x^{2}}\left[\exp \left(\frac{i x}{\xi_{G L}}\right)\right] \\
\alpha\left[\exp \left(\frac{i x}{\xi_{G L}}\right)\right]=-\frac{\hbar^{2}}{2 m^{*}}\left(\frac{i}{\xi_{G L}}\right)^{2} \exp \left(\frac{i x}{\xi_{G L}}\right) \\
-\frac{\hbar^{2}}{2 m^{*}}\left(\frac{i^{2}}{\xi_{G L}^{2}}\right) \psi=\alpha \psi
\end{gathered}
$$

This implies that,

$$
\frac{\hbar^{2}}{2 m^{*}}\left(\frac{1}{\xi_{G L(T)}^{2}}\right)=\alpha
$$

Solving for $\xi_{G L}$ at superconducting state that means, where $\alpha$ is negative yields,

$$
\xi_{G L(T)}=-\sqrt{\frac{\hbar^{2}}{2 m^{*}|\alpha|}}=\sqrt{\frac{\hbar^{2}}{2 m^{*}\left|\alpha_{0}\left(T_{c}-T\right)\right|}}
$$

where $\alpha=\alpha_{0}\left(T-T_{c}\right),-\alpha=\alpha_{0}\left(T_{c}-T\right)$ and $\xi_{G L(0)}=\sqrt{\frac{\hbar^{2}}{2 m^{*}\left|\alpha_{0}\right| T_{c}}}$

Equation (14) yields the GL coherence length [2]. Since $\alpha$ depends on temperature as $\alpha \propto\left(T-T_{c}\right)$, then we can conclude that coherence length is temperature dependent.

Now let us consider the cases:

Case (I), For superconducting state $\left(T<T_{c}\right)$,

$$
\xi_{G L(T)}=\xi_{G L(0)}\left(1-\frac{T}{T_{c}}\right)^{-\frac{1}{2}}
$$

and

Case (II), For normal state $\left(T>T_{c}\right)$,

$$
\xi_{G L(T)}=\xi_{G L(0)}\left(\frac{T}{T_{c}}-1\right)^{-\frac{1}{2}}
$$

Case (III), at $T=\mathrm{T}_{c}$, the GL theory is not valid

Where the length $\xi_{G L(0)}$ is known as the zero temperature GL coherence length.

\subsection{Calculation of Ginzburg-Landau Penetration Depth}

The surface current flows in a very thin layer of thickness $\left(\lambda_{G L}\right)$ which is called the Ginzburg-Landau penetration depth [2]. The temperature and magnetic field dependence of the penetration depth appear quite naturally in Ginzburg-Landau (GL) theory. Like the London model, the GL model is independent of the underlying mechanism for superconductivity. Ginzburg-Landau theory is strictly valid only in superconducting phase boundary and is thus not generally applicable at low temperatures [2]. In the Ginzburg-Landau theory, a complex order $\operatorname{parameter}(\psi)$ is a function of temperature, magnetic field and the spatial coordinates [5] [10]. The total free energy per unit volume of the superconducting state in the presence of a magnetic field is minimizing this expression with respect to the first GL Equation and with respect to $\boldsymbol{A}$ the current density(GL-II) Equation;

$$
F_{G L}=\frac{1}{2 m^{*}}\left|-i \hbar \nabla-\frac{e^{*}}{c} A\right|^{2} \psi+\alpha \psi+\beta|\psi|^{2} \psi=0
$$


where $m^{*}=2 m$ and $e^{*}=2 e$.

$$
|\psi|=\left|\psi_{0}\right|=\sqrt{\frac{-\alpha}{\beta}}
$$

Using Equation (17), we get the expression for current density, as follows

$$
\boldsymbol{J}_{s}=-\frac{e^{*} \hbar i}{m^{*}}\left[\psi^{*} \nabla \psi+\psi \nabla \psi^{*}\right]-\frac{e^{* 2}}{m^{*} c} \boldsymbol{A}|\psi|^{2}
$$

since $|\psi|^{2}=n_{s}=\left|\psi_{0}\right|^{2}$

$$
\boldsymbol{J}_{s}=-\frac{e^{*} \hbar i}{m^{*}}\left[\psi^{*} \nabla \psi+\psi \nabla \psi^{*}\right]-\frac{e^{* 2}}{m^{*} c} \boldsymbol{A}|\psi|^{2}
$$

Neglecting $\nabla \psi$ and $\nabla \psi^{*}$ Equation (20), becomes;

$$
\boldsymbol{J}_{s}=-\frac{e^{* 2}}{m^{*} c}|\psi|^{2} \boldsymbol{A}
$$

Using Maxwell's Equation:

$$
\nabla X \boldsymbol{B}=\frac{4 \pi}{c} \boldsymbol{J}_{s}
$$

Taking the curl on both sides of Equation (22), we get

$$
\begin{gathered}
\nabla X \nabla X \boldsymbol{B}=\frac{4 \pi}{c}\left(\nabla X \boldsymbol{J}_{s}\right) \\
\nabla(\nabla \cdot \boldsymbol{B})-\nabla^{2} \boldsymbol{B}=\frac{4 \pi}{c}\left(\nabla X \boldsymbol{J}_{s}\right)
\end{gathered}
$$

where $\nabla \cdot \boldsymbol{B}=0, \nabla X \boldsymbol{J}_{s}=-\frac{e^{* 2}}{m^{*} c}|\psi|^{2} \nabla X \boldsymbol{A}$ and $\boldsymbol{B}=\nabla X \boldsymbol{A}$

From Equation (22) and Equation (24), we get

$$
\begin{gathered}
\nabla X \boldsymbol{J}_{s}=-\frac{e^{* 2}}{m^{*} c}|\psi|^{2} \nabla X \boldsymbol{A}=-\frac{4 e^{2}}{m^{*} c}|\psi|^{2} \boldsymbol{B} \\
\nabla^{2} \boldsymbol{B}=-\frac{4 \pi}{c}\left(\nabla X \boldsymbol{J}_{s}\right)=-\frac{16 \pi e^{2}}{m^{*} c} n_{s} \boldsymbol{B}
\end{gathered}
$$

Since $|\psi|^{2}=n_{s}=\frac{-\alpha}{\beta}$ and $\lambda_{G L(T)}^{2}=\frac{m^{*} c^{2}}{16 \pi e^{2} n_{s}}$, we get

$$
\nabla^{2} \boldsymbol{B}=-\frac{4 \pi}{c}\left(\nabla X J_{s}\right)=\frac{\boldsymbol{B}}{\lambda_{G L(T)}^{2}}
$$

Therefore,

$$
\begin{gathered}
\lambda_{G L(T)}^{2}=\frac{m^{*} c^{2}}{16 \pi e^{2} n_{s}}=\frac{m^{*} c^{2} \beta}{16 \pi e^{2} \alpha} \\
\lambda_{G L(T)}=\sqrt{\frac{m^{*} c^{2} \beta}{16 \pi e^{2}|\alpha|}}=\sqrt{\frac{m^{*} c^{2}}{16 \pi e^{2} n_{s}}}=\sqrt{\frac{m c^{2}}{8 \pi e^{2} n_{s}}}
\end{gathered}
$$


where $m^{*}=2 m$ and $n_{s}=-\frac{\alpha}{\beta}=\frac{\alpha_{0}\left(T_{c}-T\right)}{\beta}$.

Therefore,

$$
\lambda_{G L(T)}=\sqrt{\frac{m c^{2} \beta}{8 \pi e^{2} \alpha_{0}\left(T_{c}-T\right)}}
$$

For

$$
\lambda_{L(0)}=\sqrt{\frac{m c^{2} \beta}{8 \pi e^{2} \alpha_{0} T_{c}}}
$$

from Equation (30) [9] it follows that, the Ginzburg-Landua penetration depth $\left(\lambda_{G L(T)}\right)$ varies as a function of temperature as:

$$
\begin{gathered}
\lambda_{G L(T)} \propto\left[1-\left(\frac{T}{T_{c}}\right)\right]^{-\frac{1}{2}} \\
\lambda_{G L(T)}=\lambda_{L(0)}\left[1-\left(\frac{T}{T_{c}}\right)^{4}\right]^{-\frac{1}{2}}
\end{gathered}
$$

where $\lambda_{L(0)}$ is the London penetration at absolute zero temperature [9].

\subsection{Calculation of the Upper Critical Magnetic Field Using Ginzburg-Landau Theory}

The upper critical magnetic field (UCMF) is the magnetic field which completely suppresses superconductivity in type-II superconductors. More properly, the UCMF is a function of temperature (and pressure) and if not specified absolute zero and standard pressure are implied. Supercondcting region nucleates spontaneously within a normal conductor when the applied magnetic field is decreased below a value denoted by $H_{c 2}$ [8]. At the onset of superconductivity, $|\psi|$ is small and we linearize the GL Equations as follows;

$$
H \psi=\frac{1}{2 m^{*}}\left(-i \hbar \nabla-\frac{e^{*}}{c} \boldsymbol{A}\right)^{2} \psi=-\alpha \psi=E \psi
$$

Since $m^{*}=2 m, e^{*}=2 e, \nabla=\frac{\partial}{\partial x} \hat{x}+\frac{\partial}{\partial y} \hat{y}+\frac{\partial}{\partial z} \hat{z}$ and $E=E_{x}+E_{y}+E_{z}$.

The upper critical magnetic field $\left(H_{c 2}\right)$ can be calculated by linearizing Equation (34) and substituting the value of $\nabla$ as:

$$
\frac{1}{2 m^{*}}\left[-i \hbar\left(\frac{\partial}{\partial x} \hat{x}+\frac{\partial}{\partial y} \hat{y}+\frac{\partial}{\partial z} \hat{z}\right)-\frac{e^{*}}{c} \boldsymbol{A}\right]^{2} \psi=-\alpha \psi
$$

The magnetic field in a superconducting region at the onset of superconductivity is just the applied field, so that $\boldsymbol{A}=\boldsymbol{B}(0, x, 0)=\boldsymbol{B} x$ and Equation (35) becomes

$$
\frac{1}{2 m^{*}}\left[-i \hbar\left(\frac{\partial}{\partial x} \hat{x}+\frac{\partial}{\partial y} \hat{y}+\frac{\partial}{\partial z} \hat{z}\right)-\frac{e^{*}}{c} \boldsymbol{B} x\right]^{2} \psi=-\alpha \psi
$$

where $\boldsymbol{P}=-i \hbar \nabla=-i \hbar\left(\frac{\partial}{\partial x} \hat{x}+\frac{\partial}{\partial y} \hat{y}+\frac{\partial}{\partial z} \hat{z}\right)$ and $\boldsymbol{P} \psi=\hbar k \psi=\hbar\left(k_{x}+k_{y}+k_{z}\right) \psi$ where the eigne value of momentum crystal is $\hbar k$. 
Therefore,

$$
\frac{1}{2 m^{*}}\left[-i \hbar\left(\frac{\partial}{\partial x}+k_{y}+k_{z}\right)-\frac{e^{*}}{c} \boldsymbol{B} x\right]^{2} \psi=-\alpha \psi
$$

Since the expression of the Hamiltonian's energy given in Equation (37) does not depend on coordinates (y and $z$ ) the corresponding momentum components $\left(k_{y}, k_{z}\right)$ are conserved.

$$
\begin{gathered}
E_{x} \psi=-\alpha \psi-\frac{\hbar^{2}}{2 m^{*}}\left(k_{y}^{2}+k_{z}^{2}\right) \psi \\
E_{x} \psi=-\left[\frac{1}{2 m^{*}}\left[-i \hbar\left(\frac{\partial}{\partial x}+\frac{\partial}{\partial y}+\frac{\partial}{\partial z}\right)-\frac{e^{*}}{c} B x\right]^{2} \psi\right]-\frac{\hbar^{2}}{2 m^{*}}\left(k_{y}^{2}+k_{z}^{2}\right) \psi \\
E_{x} \psi=\frac{\hbar^{2}}{2 m^{*}}\left(\frac{\partial^{2}}{\partial x^{2}}\right) \psi+\frac{m^{*}}{2}\left(\frac{e^{*} \boldsymbol{B} x}{m^{*} c}\right)^{2} \psi
\end{gathered}
$$

The largest value of the magnetic field $(\boldsymbol{B})$ for which the solution of Equation (40) of the lowest eigenvalue is given by

$$
E_{n}=\left(n+\frac{1}{2}\right) \hbar \omega_{c}=\left(n+\frac{1}{2}\right) \frac{\hbar e^{*} \boldsymbol{B}_{\max }}{m^{*} c}=-\alpha-\frac{\hbar^{2} k_{z}^{2}}{2 m^{*}}
$$

Let us take the smallest eigenvalues $n=0$ and $K_{z}=0$ corresponding to the highest field in which superconductivity can nucleate in the interior of a bulk sample which occurs with the upper critical magnetic field in the coefficients change of sign. From Equation (41), we have;

$$
\frac{1}{2} \hbar \omega_{c}=\frac{\hbar e^{*} \boldsymbol{B}_{\max }}{2 m^{*} c}=-\alpha
$$

where $\omega_{c}$ is the cyclotron frequency and is given by

$$
\omega_{c}=\frac{e^{*} \boldsymbol{B}_{\max }}{m^{*} c}=-\frac{2 \alpha}{\hbar}
$$

since $\boldsymbol{B}_{\max }=H_{c 2}$, solving for $H_{c 2}$, we get:

$$
H_{c 2}=-\frac{2 m^{*} c}{\hbar e^{*}}|\alpha|
$$

From the relation $\alpha=\alpha_{0}\left(T-T_{c}\right)$ that means $-\alpha=\alpha_{0}\left(T_{c}-T\right)=\frac{\hbar^{2}}{2 m^{*}}\left(\frac{1}{\xi_{G L(T)}^{2}}\right), \quad \xi_{G L(T)}=\xi_{G L(0)}\left[\left(1-\frac{T}{T_{c}}\right)\right]^{-\frac{1}{2}}$ and $\xi_{G L(T)}^{2}=\frac{\xi_{G L(0)}^{2}}{\left(1-\frac{T}{T_{c}}\right)}$

We obtain the expression of the temperature dependent upper critical magnetic field $\left(H_{c 2}\right)$,

$$
\begin{gathered}
H_{c 2}=\left(\frac{2 m^{*} c}{\hbar e^{*}}\right)\left(\frac{\hbar^{2}}{2 m^{*} \xi_{G L(0)}^{2}}\right)\left[\left(1-\frac{T}{T_{c}}\right)\right] \\
H_{c 2}=\frac{\hbar c}{e^{*} \xi_{G L(0)}^{2}}\left(1-\frac{T}{T_{c}}\right)
\end{gathered}
$$




$$
H_{c 2}=\frac{\phi_{0}}{2 \pi \xi_{G L(T)}^{2}}=\frac{\phi_{0}}{2 \pi \xi_{G L(0)}^{2}}\left(1-\frac{T}{T_{c}}\right)
$$

where $\hbar=\frac{h}{2 \pi}$ or $2 \pi \hbar=h$ and $\phi_{0}=\frac{h c}{2 e}=\frac{2 \pi \hbar c}{e^{*}}$

$$
\frac{\phi_{0}}{2 \pi}=\frac{h c}{2 \pi e^{*}}=\frac{\hbar c}{e^{*}}
$$

\subsection{Anisotropic Mass Tensor Model}

Now, consider anisotropy in mass, by introducing the effective mass tensor to the kinetic energy term of the GL Equation (3), i.e.

$$
\alpha \psi+\beta|\psi|^{2} \psi+\frac{1}{2 m^{*}}\left(-i \hbar \nabla-\frac{e^{*}}{c} A\right)^{2} \psi=0
$$

where $m^{*}$ is an effective mass tensor which is given by,

$$
m^{*}=\left[\begin{array}{ccc}
m_{x} & 0 & 0 \\
0 & m_{y} & 0 \\
0 & 0 & m_{z}
\end{array}\right]
$$

Since the coherence length $\xi_{G L(0)}$ depends on the effective mass as $\xi_{G L(T)} \propto \frac{1}{\sqrt{m}}$, the resulting Equation is formally identical with the Schrödinger Equation of a particle with charge $e^{*}=2 e$, an isotropic mass tensor $m^{*}=2 m$ in a uniform magnetic field $H$ and the energy levels that have the harmonic oscillator are given by;

$$
-\alpha=\left(n+\frac{1}{2}\right) \hbar \omega_{c}(\theta)
$$

Let us consider Newton's law of motion under the influence of lorentz force i.e.

$$
F_{l}=m^{*} \cdot \dot{v}=\frac{e^{*}}{c} v X H
$$

where $v$ is the velocity.

The upper critical magnetic field can be expressed by using cyclotron frequency with the lowest free energy;

$$
-\alpha=\frac{1}{2} \hbar \omega_{c(\theta)}
$$

The solution of upper critical magnetic field by applying elliptical orbits traversed with cyclotron frequency is given by,

$$
\omega_{c(\theta)}=\frac{\left|e^{*}\right| B_{\max }}{c}\left(\frac{\sin ^{2} \theta}{m_{x} m_{z}}+\frac{\cos ^{2} \theta}{m_{x}}\right)^{\frac{1}{2}}
$$

The solution of the lowest free energy corresponds to $n=0$ and by using Equation (49), we get

$$
\frac{1}{2} \hbar \omega_{c(\theta)}=-\alpha=\frac{1}{2} \hbar\left[\frac{\left|e^{*}\right| B_{\max }}{c}\left(\frac{\sin ^{2} \theta}{m_{x} m_{z}}+\frac{\cos ^{2} \theta}{m_{x}}\right)^{\frac{1}{2}}\right]
$$


where $\theta$ is the angle of the magnetic field that makes with the z-axis

$$
\begin{aligned}
-\alpha= & -\alpha_{0}\left(T-T_{c}\right)=\alpha_{0}\left(T_{c}-T\right) . \\
H_{c 2}= & \frac{2 c \alpha_{0}\left(T_{c}-T\right)}{\hbar e^{*}\left[\frac{\sin ^{2} \theta}{m_{x} m_{z}}+\frac{\cos ^{2} \theta}{m_{x}}\right]^{\frac{1}{2}}}
\end{aligned}
$$

From the general expression of coherence length Equation (45) we have,

$$
\begin{aligned}
& \xi_{x}=\left[\frac{\hbar^{2}}{2 m_{x} \alpha_{0}\left(T_{c}-T\right)}\right]^{\frac{1}{2}} \\
& \xi_{z}=\left[\frac{\hbar^{2}}{2 m_{z} \alpha_{0}\left(T_{c}-T\right)}\right]^{\frac{1}{2}}
\end{aligned}
$$

Using the expression for the flux quantization, $\phi_{0}=\frac{h c}{\left|e^{*}\right|}$ and Equation (45), $H_{c 2}$ can be expressed as,

$$
H_{c 2}=\frac{\phi_{0}}{2 \pi\left[\frac{\sin ^{2} \theta}{\xi_{x}^{2} \xi_{z}^{2}}+\frac{\cos ^{2} \theta}{\xi_{x}^{4}}\right]^{\frac{1}{2}}}
$$

For fields parallel and perpendicular to the symmetry plane we can write Equation (53) as:

$$
H_{c 2} \|=\frac{\phi_{0}}{2 \pi \xi_{z} \xi_{z}}
$$

and

$$
H_{c 2} \perp=\frac{\phi_{0}}{2 \pi \xi_{x}^{2}}
$$

Equations (54) and (55) are the mathematical expressions of the upper critical magnetic field $\left(H_{c 2}\right)$ for fields parallel and perpendicular to the symmetry axis [4].

\section{Results and Discussion}

From Equation (15), we obtained the graph that shows the relationship between the GL coherence length and temperature $(\mathrm{T})$ as indicated in Figure 1.

As can be see from Figure 1, the GL coherence length increases with temperature and diverge as $T \rightarrow T_{c}$. $\xi_{G L(0)}$ has the same value with the BCS coherence length i.e. $\left(\xi_{G L(0)}=\xi_{0}\right)[2]$.

We have already calculated the GL penetration depth in (33), the relationship between the GL penetration depth and temperature $(\mathrm{T})$ is shown in Figure 2.

From Figure 2, we observe that, the increase of the GL penetration depth with temperature (T) and generally, we observe that, the penetration depth rises asymptotically as the temperature approaches $T_{c}$. Thus, the penetration of field increase as the temperature approaches to $T_{c}$.

We finally determined the expression for upper critical magnetic field for superconducting $\mathrm{HoMo}_{6} \mathrm{Se}_{8}$ using the GL Equation (45) and by taking experimental data and upper critical magnetic fields for parallel and perpendicular, we plot the upper critical fields at parallel and perpendicular to the symmetry axis as shown in Figure 3 [6] [7]. 


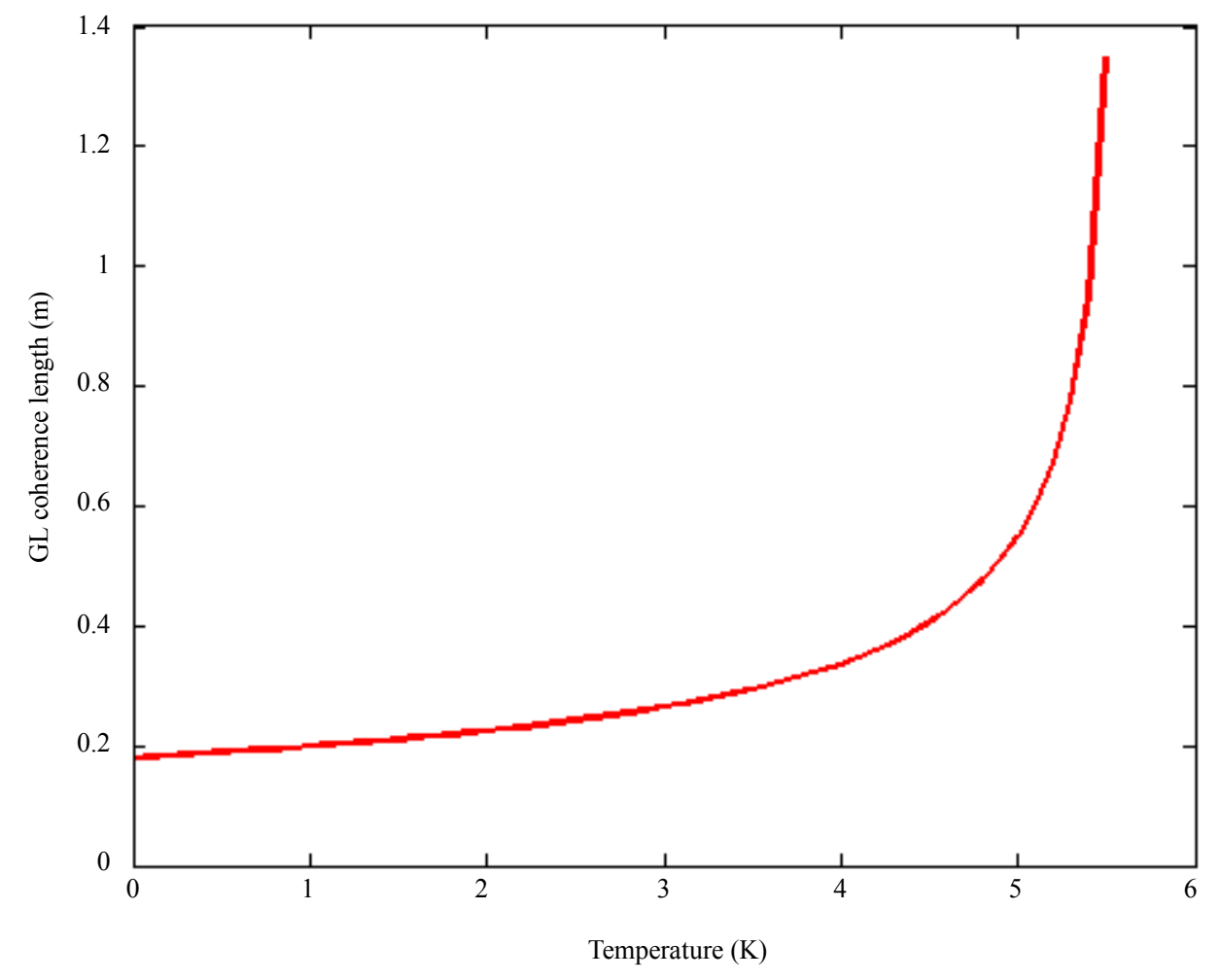

Figure 1. GL coherence length versus temperature (T).

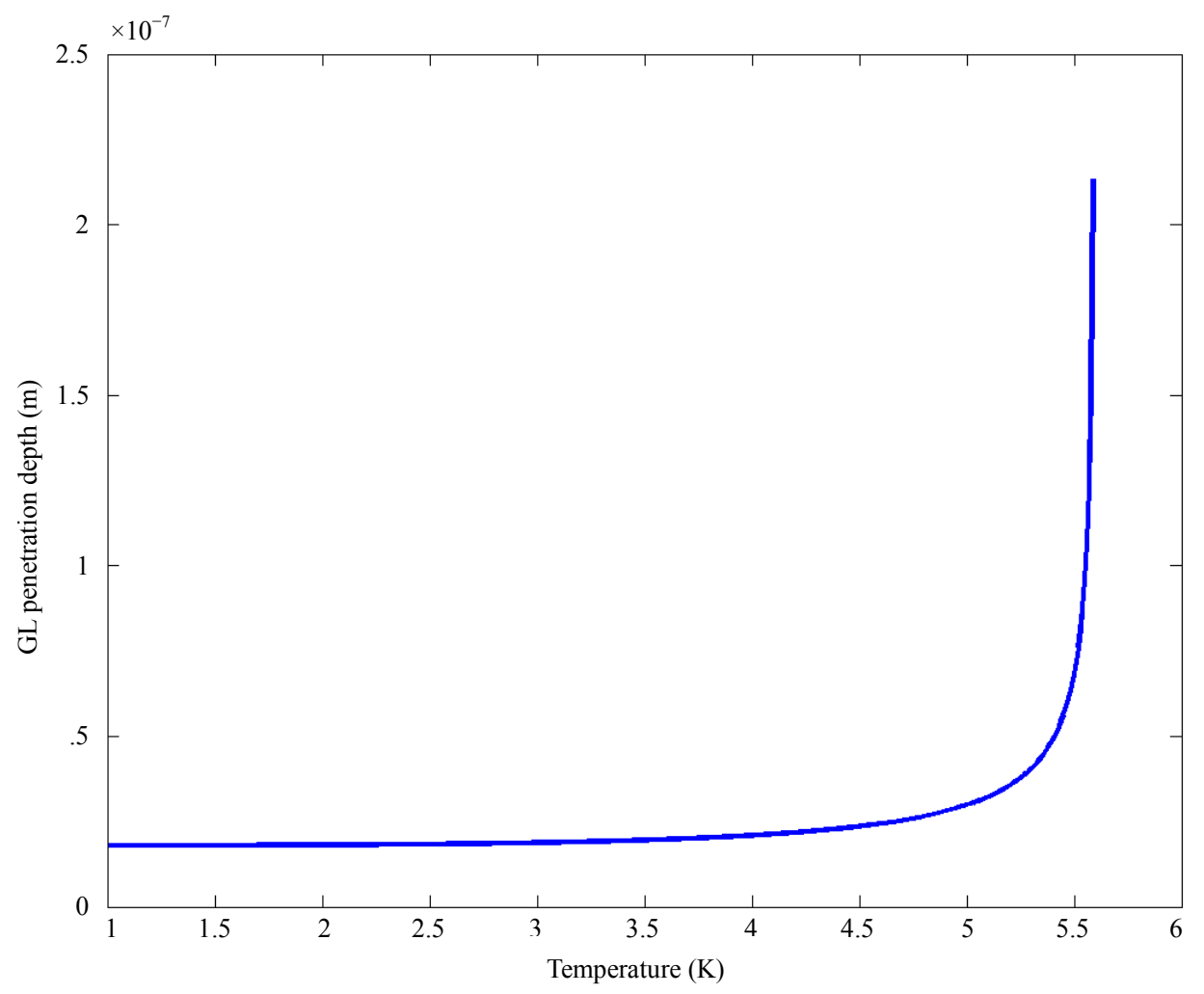

Figure 2. GL penetration depth versus temperature (T). 


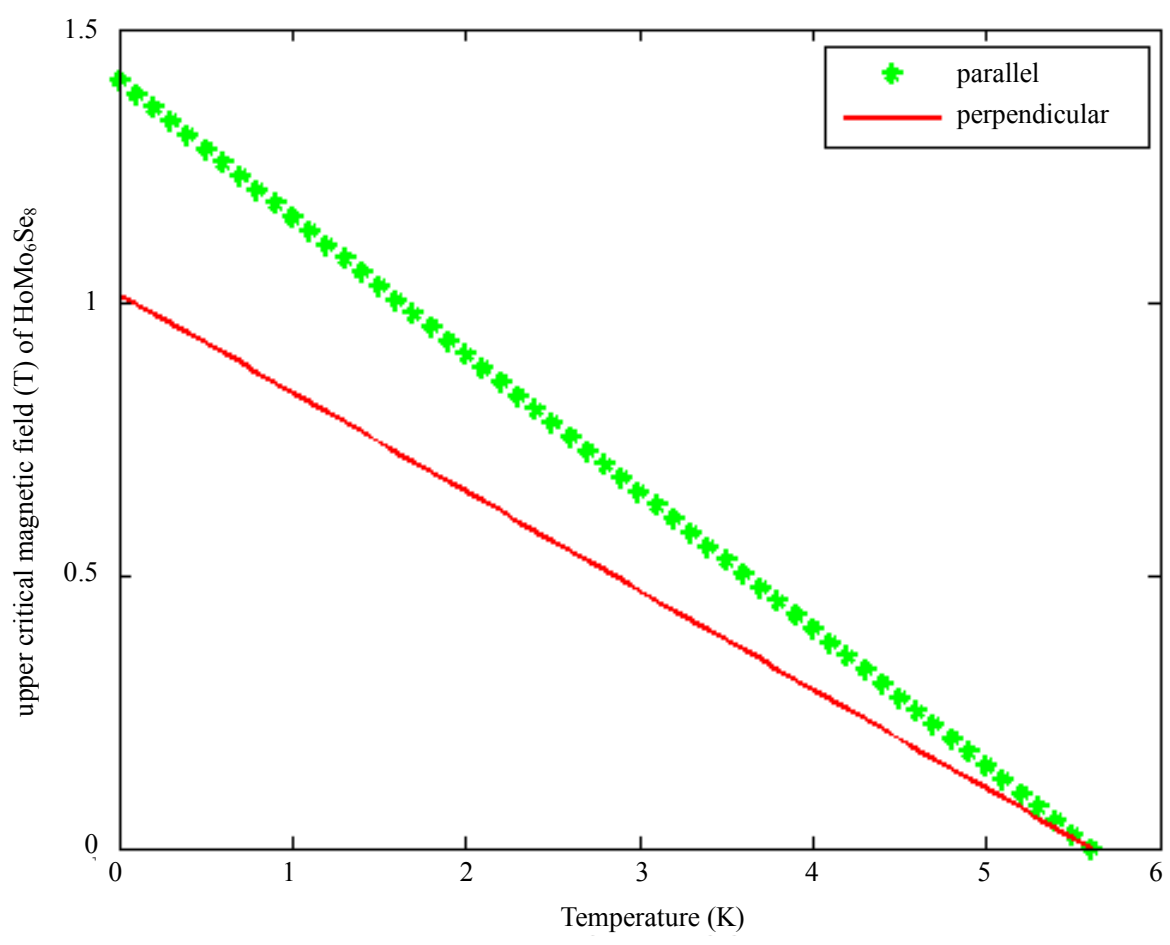

Figure 3. Upper critical magnetic field parallel and perpendicular to the symmetry axis versus temperature $(\mathrm{T})$.

From Figure 3, we can see that, the upper critical magnetic field decreases as temperature increases and reaches to zero at the critical temperature of superconducting $\mathrm{HoMo}_{6} \mathrm{Se}_{8}$, which agrees with the experimental observations [6]. And as can be seen from $\$$, the upper critical magnetic field $\left(H_{c 2}\right)$ parallel and perpendicular to the symmetry axis of superconducting $\mathrm{HoMo}_{6} \mathrm{Se}_{8}$ is inversely proportional to the GL coherence length and fits.

\section{Conclusion}

The aim of this research is to determine the upper critical field of superconducting $\mathrm{HoMo}_{6} \mathrm{Se}_{8}$ by using Ginzburg-Landau approach. From the calculation, the effect of coherence length, penetration depth and anisotropy in mass tensor on upper critical field are considered in our model. And finally figures are plotted by using MATLAB scripts. From the figures plotted, it can be concluded that the upper critical magnetic field of superconducting $\mathrm{HoMo}_{6} \mathrm{Se}_{8}$ is inversely related to temperature which is in agreement with experimental observations $[6]$.

\section{References}

[1] Owens, F.J. and Poole, Jr., C.P. (2002) The New Superconductors. Kluwer Academic Publishers, New York.

[2] Mourachkine, A. (2004) Room Temperature Superconductivity. University of Cambridge, Cambridge.

[3] Patterson, J.D. and Bailey, B.C. (2010) Solid-State Physics, Introduction to the Theory.

[4] Kittel, C. (2005) Introduction to Solid State Physics. John Wiley and Sons, Inc., Hoboken.

[5] Prestemon, S. and Ferracin, P. (2007) Basics of Superconductivity. IEEE Transactions on Applied Superconductivity, 3, 4.

[6] Lynn, J.W., Gotaas, J.A., Erwin, R.W., Ferrrell, R.A., Bhattacharjee, J.K., Shelton, R.N. and Klavins, P. (1984) Temperature Dependent Sinusoidal Magnetic Order in the Superconductor HoMo ${ }_{6} \mathrm{Se}_{8}$. Physical Review Letters, 52, 133.

[7] Gotaas, J.A. and Lynn, J.W. (1986) Magnetic Field Dependence of the Small Angle Neutron Scattering in $\mathrm{HoMo}_{6} \mathrm{Se}_{8}$. Journal of Magnetism and Magnetic Materials, 54, 1529-1530. http://dx.doi.org/10.1016/0304-8853(86)90915-7

[8] Leggett, A.J. (1975) A Theoretical Description of the New Phases of Liquid ${ }^{3}$ He. Reviews of Modern Physics, $47,331$. http://dx.doi.org/10.1103/RevModPhys.47.331 
[9] Maki, K. and Tsuneto, T. (1964) Pauli Paramagnetism and Superconducting State. Progress of Theoretical Physics, 31, 945. http://dx.doi.org/10.1143/PTP.31.945

[10] Antoine, J.-P., Govaerts, J., Peeters, F., Gerard, J.-M., Gregoire, G., Piraux, L. and Ruelle, P. (2005) A Relativistic BCS Theory of Superconductivity Juillet.

[11] Bulaevskii, L.N., Ginzburg, V.L. and Sobyanin, A.A. (1988) The Macroscopic Theory of Superconductors with a Short Coherence Length. Journal of Experimental and Theoretical Physics (JETP), 94, 355. 